\title{
Quantitative evaluation of diffusion- weighted imaging with multiple b-values in vertebral fractures
}

\section{Fatma Kulali}

Radiology Department, University of Health Sciences, Umraniye Training and Research Hospital, Istanbul, Turkey
Received: 2018-08-16

Accepted: 2018-09-22

UDC: 616.1

\author{
J Clin Med Kaz 2018;3(49):35-41 \\ Corresponding Author: Fatma Kulali, MD. Postal \\ address: Adem Yavuz street. No:1, University of Health \\ Sciences, Umraniye Training and Research Hospital, \\ Department of Radiology, Umraniye, 34764, Istanbul, \\ Turkey. Phone: +90 (216) 632 1818*1462; Fax: +90 \\ (216) 6327124 \\ E-mail: ftkulali@gmail.com
}

\section{Abstract}

Aim: Differentiating benign from malignant vertebral fracture is sometimes difficult in geriatric oncology patients. Accurate diagnosis is necessary for treatment planning. Therefore, we aimed to investigate the role of quantitative evaluation of diffusion-weighted imaging (DWI) at multiple b-values of 200, 400 and $600 \mathrm{~s} / \mathrm{mm} 2$ in differentiating benign from malignant thoracolumbar vertebral fractures and to determine an optimal b-value.

Methods: Forty-four patients with 72 vertebral fractures were enrolled. Magnetic resonance imaging (MRI) findings combined with DWI at b-values of 200, 400 and $600 \mathrm{~s} / \mathrm{mm} 2$ were evaluated. Apparent diffusion coefficient (ADC) and normalized ADC values were obtained. Radiological and histopathological/ follow-up results were compared.

Results: Of 72 vertebral fractures, 22 were benign and 50 were malignant. Mean ADC and normalized ADC values of malignant group were lower than benign group's in all $b$-values $(p<0.05)$. Despite of no significant difference between ADC values at b-values of 200, 400 and $600 \mathrm{~s} / \mathrm{mm} 2$ within each group, normalized ADC values were lower at b-value of $200 \mathrm{~s} / \mathrm{mm} 2$ than those of at 600 $\mathrm{s} / \mathrm{mm} 2$ in malignant group $(\mathrm{p}<0.05)$.

Conclusion: MRI combined with DWI is a problem solving modality especially in geriatric oncology patients. Performing DWI at b-value of 200 $\mathrm{s} / \mathrm{mm} 2$ and estimation of normalized ADC value for optimization of data are recommended.

Key words: apparent diffusion coefficient, diffusion-weighted imaging, magnetic resonance imaging, vertebral fracture

\section{ОМЫРТҚАНЫН СЫНЫҚТАРЫ КЕЗІНДЕГІ КӨПТЕГЕН В-МӘНІМЕН ДИФФУЗИЯЛЫҚ-ӨЛШЕНГЕН ВИЗУАЛИЗА- ЦИЯЛАУДЫ САНДЫҚ БАҒАЛАУ}

Фатма Кулали

Радиологиялық бөлімі, Медицина ғылымдары университеті, Умрание білім беру және ғылыми-зерттеу клиникасы, Түркия

\section{ТҰЖЫРЫМДАМА}

Мақсаты: Кейде гериатриялық онкологиялық науқастарда омыртқаның сынуы кезінде омыртқа сүйектерінің қатерсіз ісіктерін қатерлі ісіктерден ерекшелеу қиынға соғады. Сондықтан, біздің мақсатымыз - 200, 400 және 600 c/мм2 көптеген b-мәнін дифффузиялық-өлшенген визуализациялауды сандық бақылаудың рөлін зерделеу және омыртқаның кеуде-белінің сынықтары барысында қатерлі ісіктерден қатерсіз ісікті дифференциациялау үшін тиімді b-мәнін айқындау болып табылады.

Әдістері: Зерттеуге 72 омыртқа сынықтарымен 44 пациент қатысты. 200, 400 және 600 c/мм2 b-мәні кезінде диффрузиялық-өлшенген визуализациялаумен үйлестікте магниттік-резонанстық томографияның нәтижелері бағаланды. Диффрузияның өлшенетін коэффициентінің мәні және дифффузияның өлшенетін коэфффициентінің нормаланған мәндері алынды. Радиологиялық және гистологиялық қадағалаудың нәтижелері салыстырылды.

Нәтижелері: 72 омыртқа сынықтарынан 22-уі омыртқа сүйектерінің қатерсіз ісігі және 50-і қатерлі ісік болып шықты. Қатерлі ісіктер тобының диффузиясының өлшенетін коэфффициентінің орташа нормаланған мәндері 200 c/мм2b-мәні кезінде қатерсіз ісіктер тобына, қатерлі ісіктер тобында 600 с/мм2 қарағанда, төмен болды.

қорытынды: магниттік-резонанстық томография диффрузиялық-өлшенген визуализациялаумен бірге - бұл әсіресе, гериатриялық онкологиялық науқастардың проблемаларын шешу әдісі. 200 c/мм2 b-мәні кезінде дифффузиялық-өлшенген визуализациялауды өткізуге және деректерді оңтайландыру үшін диффузиялау өлшенетін коэфициентінің нормаланған мәнін бағалау ұсынылады.

Негізгі сөздер: диффузияның өлшенетін коэффициенті, диффузиялық-өлшенетін визуализациялау, магниттік-резонанстық томография, омыртқаның сынуы 


\section{КОЛИЧЕСТВЕННАЯ ОЦЕНКА ДИФФУЗИОННО-ВЗВЕШЕННОЙ ВИЗУАЛИЗАЦИИ С МНОЖЕСТВЕННЫМ В-ЗНАЧЕНИЕМ ПРИ ПЕРЕЛОМАХ ПОЗВОНКОВ}

Фатма Кулали

Радиологическое отделение, Университет медицинских наук, Образовательная и научно-исследовательская клиника Умрание, Стамбул, Турция

\section{PEЗЮME}

Цель: Иногда сложно отличить доброкачественную опухоль костей позвоночника от злокачественной при переломе позвоночника у гериатрических онкобольных. Необходим точный диагноз для планирования лечения. Поэтому, нашей целью является изучить роль количественной оценки дифффузионно-взвешенной визуализации множественных b-значений в 200, 400 и 600 с/мм2 и определить оптимальное b-значение для дифференциации доброкачественной опухоли от злокачественной при грудопоясничных переломах позвоночника.

Методы: В исследовании приняли участие сорок четыре пациента с 72 переломами позвоночника. Оценивались результаты магнитнорезонансной томографии в сочетании с диффузионно-взвешенной визуализацией при b-значениях в 200,400 и 600 с/мм2. Получены значения измеряемого коэффициента диффузии и нормализованные значения измеряемого коэффрициента дифффузии. Сравнивались результаты радиологического и гистологического наблюдения.

Результаты: Из 72 переломов позвоночника, 22 оказалисьдоброкачественными опухолями костей позвоночникаи 50 злокачественными. Средние и нормализованные значения измеряемого коэффициента диффрузии группы злокачественной опухоли были ниже, чем в группе доброкачественной опухоли при b-значении в 200 c/мм2, чем при 600 c/мм2 в группе злокачественной опухоли (p<0.05).

Заключение: Магнитно-резонансная томография совместно с дифффузионно-взвешенной визуализацией это метод решения проблемы в особенности у гериатрических онкобольных. Рекомендуется проведение диффузионно-взвешенной визуализации при b-значении в 200 c/ мм2 и оценка нормализованного значения измеряемого коэффициента диффузии для оптимизации данных.

Ключевые слова: Измеряемый коэфффициент диффузии, дифффузионно-взвешенная визуализация, магнитно-резонансная томография, перелом позвоночника

\section{Introduction}

Vertebral fractures are frequently seen due to osteoporosis, trauma and tumor. Incidence of vertebral fractures continues to increase with age [1-5]. The diagnosis and determining the etiology of vertebral fracture are generally made on the basis of history and clinical findings combined with radiological imaging such as conventional radiography, computed tomography (CT) and magnetic resonance imaging (MRI). MRI provides high tissue contrast resolution. Therefore, bone marrow edema, associated soft tissue component and contrast enhancement can accurately be detected on MRI [1-5]. However, some acute osteoporotic and traumatic vertebral fractures can mimic malignant vertebral fractures with increased contrast enhancement and high signal intensity on T2-weighted sequences due to edema and inflammatory reactions. Differentiating benign from malignant vertebral fracture is very important especially in geriatric oncology patients. Existence of bone metastasis changes the management of patient. In patients with bone metastases, conservative treatment is preferred because of reduced probability of being cured. In some patients, additional imaging modality is required for differential diagnosis [1-5].

To the best of our knowledge, quantitative evaluation of diffusion-weighted imaging (DWI) at b-values of 200, 400 and $600 \mathrm{~s} / \mathrm{mm} 2$ in differential diagnosis of benign and malignant thoracolumbar vertebral fractures has not been investigated before. For this reason, aims of this study were to investigate the role of quantitative evaluation of DWI at multiple b-values in differentiating benign from malignant thoracolumbar vertebral fractures and to determine an optimal b-value for differential diagnosis.

\section{Materials and Methods}

The Institutional Review Board approved this retrospective study, and informed consent was waived. Imaging reports of thoracolomber MRI examinations in hospital information system (HIS) were searched retrospectively at a single institution between March 2003 and March 2005. The terms used were vertebral fractures, vertebral tumor, metastasis, lymphoma, multiple myeloma, cancer and carcinoma. There were $51 \mathrm{MRI}$ examinations of consecutive patients. Then, medical imaging records of these patients were reviewed from picture archiving and communications system (PACS). MRI examinations without
DWI ( $\mathrm{n}=5)$ and two patients with insufficient images due to artefacts were excluded. A total 44 patients with 72 vertebral fractures who had undergone thoracolomber MRI combined with DWI were enrolled in this study. There were 22 women and 22 men with a mean age of $45 \pm 18$ (SD) years (range: 19-78 years).

MRI examinations were performed on a 1.5 Tesla system (GyroscanIntera, Philips Medical Systems, Best, the Netherlands). MRI sequences were as follows; sagittal T2weighted turbo spin-echo (TSE) (TR/TE: 3500/120, field of view FOV: $325 \mathrm{~mm}$, matrix: 264x512, slice thickness $5 \mathrm{~mm}$ ), sagittal T1-weighted TSE (TR/TE: 400/11, field of view FOV: $325 \mathrm{~mm}$, matrix: 264x512, slice thickness $5 \mathrm{~mm}$ ), sagittal T2weighted TSE spectral presaturation with inversion recovery (SPIR) (TR/TE: 3500/120, field of view FOV: 325 mm, matrix: 216x512, slice thickness $5 \mathrm{~mm}$ ), axial T2-weighted TSE (TR/ TE: 3500/120, FOV: 225mm, matrix: 213x512, slice thickness $5 \mathrm{~mm}$,), sagittal pre-contrast and post-contrast T1-weighted TSESPIR (TR/TE: 400/11, FOV: 325mm, matrix: 264x512, slice thickness $5 \mathrm{~mm}$ ) sequences, sagittal diffusion weighted single-shot echo-planar imaging (EPI) (TR/TE: 1839/86, EPI factor: 77 , FOV: $270 \mathrm{~mm}$, image matrix: $77 \times 256$, slice thickness $5 \mathrm{~mm}$ ) with b-values $0,200,400$ and $600 \mathrm{~s} / \mathrm{mm} 2$.

An experienced radiologist (FK) evaluated the MRI and DWI findings of thoracolumbar vertebral fractures without knowing the history, clinical or surgical results of patients. Signal intensity on T1- and T2-weighted images, convexity of posterior vertebral corpus wall, presence of contrast enhancement, involvement of posterior vertebral elements and associated soft tissue component on MRI were noted. Low signal intensity on T1-weighted sequence and high signal intensity on T2-weighted sequence without contrast enhancement was defined as edema.

Region of interest (ROI) measurements in vertebral fracture and adjacent normal vertebral bone marrow were performed at three different locations inside vertebral fracture in the dedicated workstation (Philips Medical Systems). The measurements of circular ROIs ranged in size between $110 \mathrm{~mm} 2$ and $130 \mathrm{~mm} 2$. For each vertebral fracture $(n=72)$ and adjacent normal vertebral bone marrow $(n=72)$, the mean value of ROI measurements was estimated on ADC map.The normalized ADC value (ratio of mean $\mathrm{ADC}$ value in vertebral fracture to mean $\mathrm{ADC}$ value in adjacent normal vertebral bone marrow) was estimated. 
Vertebral fractures were classified into two groups as benign and malignant according to follow-up and histopathological results. Vertebral fractures without clinical and radiological progression at least 6 months of follow-up were grouped as benign. All malignant vertebral fractures were histopathologically proven.

Radiological and histopathological/follow-up findings were compared. Kruskal-Wallis test or Mann-Whitney U test were used, where appropriate, and $\mathrm{p}<0.05$ was used to determine statistical significance. The diagnostic capabilities of DWI for differentiating malignant from benign vertebral fractures were analyzed by estimation of sensitivity, specificity, positive predictive value (PPV), negative predictive value (NPV) and accuracy ratios. Statistical analysis was done by using MedCalc statistical software 12.1.4.0

\section{Results}

Forty-four patients with 72 thoracolumbar vertebral fractures were investigated. Of 72 vertebral fractures, 22 were benign and 50 were malignant. Mean follow-up period of benign thoracolumbar vertebral fractures was $13.27 \pm 5.38$ months (range: 6-24 months). Among benign $(n=22)$ vertebral fractures, 10 were osteoporotic and 12 were traumatic fractures (Figure 1). Of 50 malignant vertebral fractures, there were multiple myeloma $(n=1)$, metastases of invasive ductal breast cancer $(n=6)$, prostate adenocancer $(n=15)$, thyroid papillary cancer $(n=18)$, non-small cell lung cancer $(n=5)$, Ewing sarcoma $(n=2)$ and lymphoma $(n=3)$. Associated soft tissue component $(7 / 50,14 \%)$ and involvement of posterior elements $(11 / 50$, $22 \%$ ) were only seen in malignant fractures (Figure 2). Among 22 benign (osteoporotic and traumatic) fractures, 4 had contrast enhancement. Some qualitative MRI findings such as low signal intensity on T1-weighted sequence, high signal intensity on T2weighted SPIR sequence, total vertebral corpus involvement, convexity of posterior vertebral corpus wall and contrast enhancement were more frequent in our malignant group. Associated soft tissue component and involvement of posterior

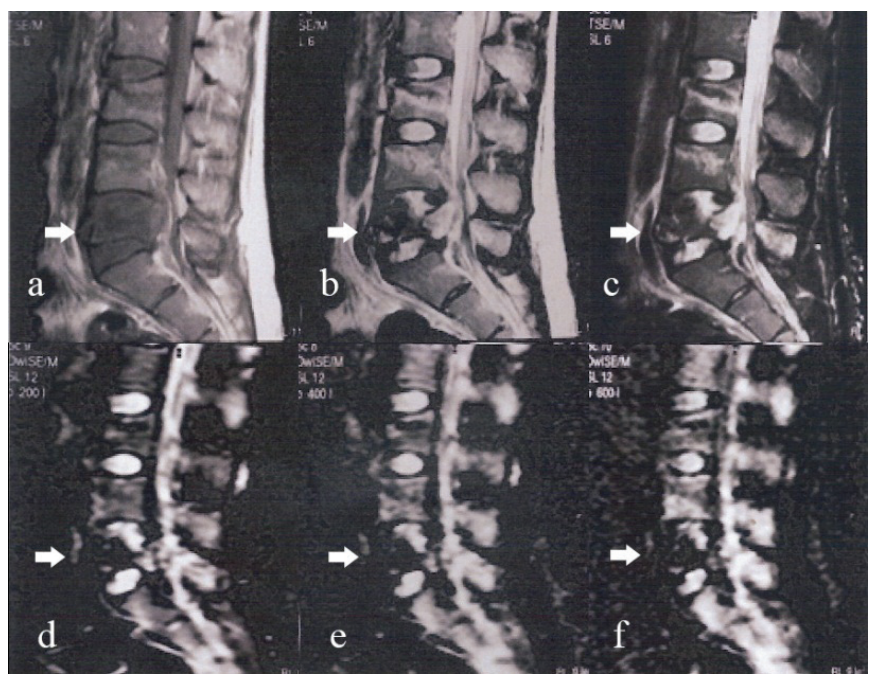

Figure 1. - 19-year-old woman who had traumatic L3, L4 and L5 (arrow) vertebral fractures with bone marrow edema on sagittal T1-weighted sequence (a), on sagittal T2-weighted sequence (b), on sagittal T2-weighted SPIR sequence (c), low signal intensities of fractures and high signal intensities of bone marrow edema on sagittal DWI at b value of 200 (d), 400 (e) and $600 \mathrm{~s} / \mathrm{mm} 2$ (f). vertebral elements were not seen in our benign group. Of 50 malignant vertebral fractures, 3 showed low signal intensity on DWI. Of 22 benign fractures, 2 had high signal intensity on DWI and low signal intensity on ADC map. MRI combined with DWI characteristics of benign and malignant vertebral fractures are shown in Table 1.

The sensitivity, specificity, positive predictive value (PPV), negative predictive value (NPV), and accuracy of qualitative DWI findings for diagnosing malignant vertebral fractures was $94 \%, 91 \%, 96 \%, 87 \%$, and $93 \%$, respectively.

Mean ADC value of normal adjacent vertebral bone marrow with $0.39 \pm 0.07(0.37-0.41) \times 10-3 \mathrm{~mm} 2 / \mathrm{s}$ at b-value of $200 \mathrm{~s} / \mathrm{mm} 2$ was significantly lower than those of malignant and benign vertebral fractures $(p<0.05)$. Maximum mean ADC value of malignant vertebral fractures was $1.92 \times 10-3 \mathrm{~mm} 2$ $/ \mathrm{s}$ and minimum mean ADC value of benign fractures was $2.68 \times 10-3 \mathrm{~mm} 2 / \mathrm{s}$ at b-value of $200 \mathrm{~s} / \mathrm{mm} 2$. At b-values of 200 , 400 and 600 , mean ADC values of malignant vertebral fractures were found statistically lower than benign vertebral fractures' $(p<0.05)$. However, there was no significant difference between mean ADC values at b-values of 200, 400 and $600 \mathrm{~s} / \mathrm{mm} 2$ within each group $(\mathrm{p}>0.05)$. But, normalized ADC values were found significantly lower at b-value of $200 \mathrm{~s} / \mathrm{mm} 2$ than those of at $\mathrm{b}$-value of $600 \mathrm{~s} / \mathrm{mm} 2$ in malignant group $(\mathrm{p}<0.05)$. Normalized $\mathrm{ADC}$ values of benign group was also significantly higher than that of malignant group in all $b$-values $(p<0.05)$. Mean ADC and normalized ADC values of benign and malignant vertebral fractures are demonstrated in Table 2.

\section{Discussion}

MRI is an appropriate method to detect bone marrow edema or tumor. Sometimes, additional imaging modalities are needed to make accurate diagnosis because of overlapping MRI findings [1-7].

DWI indicates random movement of water molecules in tissue. Apparent diffusion coefficient (ADC) map is obtained from DWI automatically and also provides opportunity of

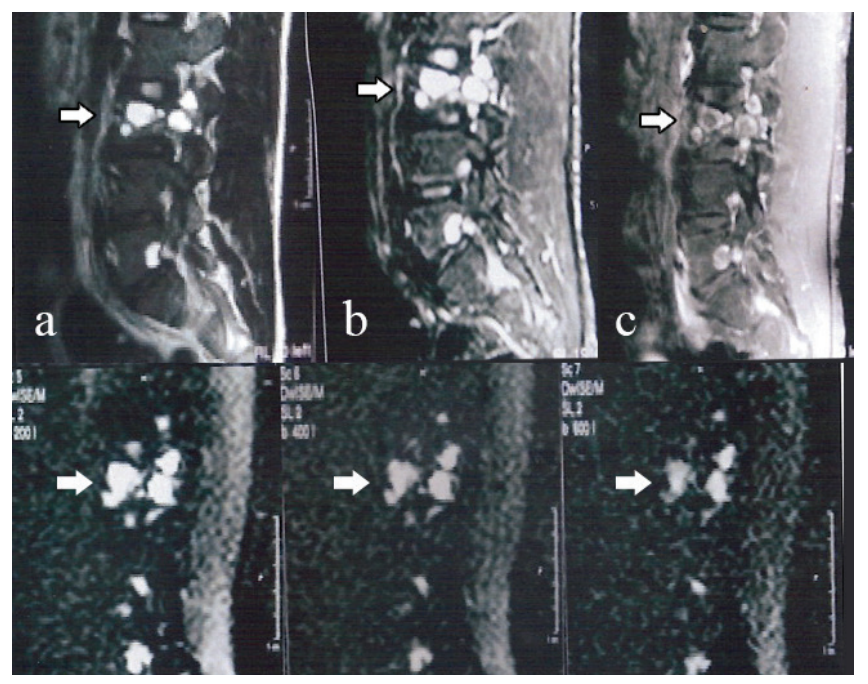

Figure 2. 74-year-old man with prostate adenocancer had metastatic vertebral fractures at L3 (arrow) and L5 vertebra which show low signal intensity on sagittal T1-weighted sequence (a), high signal intensity on sagittal T2-weighted SPIR sequence (b), contrast enhancement on sagittal postcontrast T1-weighted SPIR sequence (c) and high signal intensity on sagittal DWI at b value of 200 (d), 400 (e) and 600 $\mathrm{s} / \mathrm{mm} 2(\mathrm{f})$. 
Table 1 Distribution of qualitative and quantitative characteristics of MRI combined with DWI in benign and malignant vertebral fractures

\begin{tabular}{|c|c|c|c|c|c|c|c|c|c|c|c|c|c|c|c|c|c|}
\hline & & & & & & High SIon & Low SI & & & & & & & NADCr & & & \\
\hline P.Noa & Age & $\mathrm{Gb}$ & $\mathrm{Me} / \mathrm{Bf}$ & $\mathrm{Fx}(\mathrm{n}) \mathrm{g}$ & Etiology & T2-WIh & T2-SPIR1 & T1-WIj & CEk & $\mathrm{BEl}$ & PEm & TCinvn & Convo & STp & 200 & 400 & 600 \\
\hline 1 & 55 & $\mathrm{Fc}$ & $\mathrm{B}$ & 1 & OPs & 1 & 1 & 1 & No & 1 & No & No & No & No & 12.08 & 12.08 & 12.08 \\
\hline 2 & 33 & $\mathrm{Md}$ & $\mathrm{B}$ & 2 & Trauma & 2 & No & No & No & No & No & 1 & 1 & No & 15.46 & 11.17 & 15.46 \\
\hline 3 & 57 & $\mathrm{~F}$ & $\mathrm{~B}$ & 1 & $\mathrm{OP}$ & 1 & 1 & 1 & No & 1 & No & No & 1 & No & 7.63 & 8.75 & 7.63 \\
\hline \multirow[t]{2}{*}{4} & 52 & M & $\mathrm{B}$ & 2 & Trauma & 2 & No & 2 & No & 1 & No & 1 & 1 & No & 11.59 & 10.57 & 11.59 \\
\hline & & & & & & & & & & & & & & & 21.88 & 12.62 & 21.88 \\
\hline 5 & 27 & $\mathrm{M}$ & $\mathrm{B}$ & 1 & Trauma & 1 & No & No & No & No & No & No & 1 & No & 14.80 & 11.58 & 14.80 \\
\hline \multirow[t]{2}{*}{6} & 19 & $\mathrm{M}$ & $\mathrm{B}$ & 2 & Trauma & 2 & No & No & No & No & No & No & 1 & No & 20.27 & 18 & 19.58 \\
\hline & & & & & & & & & & & & & & & 11.51 & 10.95 & 11.93 \\
\hline \multirow[t]{4}{*}{7} & 65 & $\mathrm{~F}$ & $\mathrm{~B}$ & 4 & $\mathrm{OP}$ & No & No & No & 4 & No & No & 1 & 1 & No & 7.68 & 7.68 & 7.97 \\
\hline & & & & & & & & & & & & & & & 12.17 & 12.17 & 12.17 \\
\hline & & & & & & & & & & & & & & & 8.12 & 9.93 & 8.12 \\
\hline & & & & & & & & & & & & & & & 8.37 & 8.88 & 8.37 \\
\hline \multirow[t]{2}{*}{8} & 21 & $\mathrm{M}$ & $\mathrm{B}$ & 2 & Trauma & No & No & No & No & No & No & No & No & No & 13.59 & 13.59 & 13.22 \\
\hline & & & & & & & & & & & & & & & 12.94 & 10.16 & 12.38 \\
\hline 9 & 73 & $\mathrm{M}$ & $\mathrm{B}$ & 2 & OP & 1 & No & 1 & No & No & No & 1 & 1 & No & 13.27 & 11.68 & 13.27 \\
\hline & & & & & & & & & & & & & & & 11.61 & 9.48 & 11.61 \\
\hline 10 & 19 & $\mathrm{~F}$ & $\mathrm{~B}$ & 3 & Trauma & 3 & 3 & 3 & No & 2 & No & No & 1 & No & 9.80 & 9.80 & 9.57 \\
\hline & & & & & & & & & & & & & & & 14.21 & 11.78 & 13.60 \\
\hline & & & & & & & & & & & & & & & 16.62 & 16.62 & 15.58 \\
\hline 11 & 61 & $\mathrm{~F}$ & $\mathrm{~B}$ & 2 & OP & 2 & No & 1 & No & 1 & No & 1 & 1 & No & 18 & 16.70 & 18.64 \\
\hline & & & & & & & & & & & & & & & 9.73 & 9.73 & 9.14 \\
\hline 12 & 70 & $\mathrm{M}$ & $\mathrm{M}$ & 4 & Prostate ca & 4 & 4 & 4 & 4 & No & No & 3 & No & No & 3.58 & 4.13 & 4.41 \\
\hline & & & & & & & & & & & & & & & 3.81 & 2.48 & 4.47 \\
\hline & & & & & & & & & & & & & & & 3.30 & 4.13 & 4.13 \\
\hline & & & & & & & & & & & & & & & 4.18 & 3.57 & 4.69 \\
\hline 13 & 78 & $\mathrm{M}$ & $\mathrm{M}$ & 1 & Prostate ca & 1 & 1 & 1 & 1 & No & 1 & No & No & No & 3.81 & 3.81 & 3.81 \\
\hline 14 & 43 & $\mathrm{M}$ & $\mathrm{M}$ & 2 & Thyroid ca & 1 & 2 & 1 & 1 & 1 & 1 & No & 1 & 2 & 2.18 & 2.18 & 2.18 \\
\hline & & & & & & & & & & & & & & & 1.74 & 1.74 & 1.74 \\
\hline 15 & 21 & $\mathrm{M}$ & $\mathrm{M}$ & 2 & Ewing S. & 2 & 2 & 2 & 2 & No & 1 & 1 & 1 & 1 & 3.91 & 3.91 & 4.04 \\
\hline & & & & & & & & & & & & & & & 3.93 & 4.14 & 4.14 \\
\hline 16 & 34 & $\mathrm{~F}$ & $\mathrm{M}$ & 1 & Thyroid ca & 1 & 1 & 1 & 1 & No & No & 1 & 1 & No & 1.62 & 1.62 & 1.62 \\
\hline 17 & 41 & $\mathrm{M}$ & $\mathrm{M}$ & 1 & Lung ca & 1 & 1 & 1 & 1 & No & No & 1 & 1 & No & 2.25 & 1.89 & 1.89 \\
\hline 18 & 78 & M & $\mathrm{M}$ & 1 & MM & No & 1 & 1 & 1 & No & No & No & No & No & 3.78 & 4.09 & 4.39 \\
\hline 19 & 40 & $\mathrm{~F}$ & $\mathrm{M}$ & 1 & Breast ca & No & 1 & 1 & 1 & No & No & 1 & 1 & No & 3.91 & 4.35 & 4.35 \\
\hline 20 & 41 & $\mathrm{~F}$ & $\mathrm{M}$ & 2 & Thyroid ca & 1 & 2 & 1 & 1 & No & 1 & 1 & 1 & 2 & 2.03 & 2.03 & 2.03 \\
\hline & & & & & & & & & & & & & & & 1.50 & 1.50 & 1.50 \\
\hline 21 & 42 & $\mathrm{M}$ & $\mathrm{M}$ & 1 & Lymphoma & 1 & 1 & 1 & 1 & No & No & No & No & No & 4.18 & 4.40 & 4.52 \\
\hline 22 & 35 & $\mathrm{~F}$ & $\mathrm{M}$ & 1 & Thyroid ca & 1 & 1 & 1 & 1 & No & 1 & No & No & 1 & 3.72 & 3.93 & 3.93 \\
\hline 23 & 40 & $\mathrm{~F}$ & M & 1 & Thyroid ca & 1 & 1 & 1 & 1 & 1 & No & No & No & No & 2.82 & 3.04 & 3.48 \\
\hline 24 & 73 & M & M & 1 & Lung ca & 1 & 1 & 1 & 1 & No & 1 & No & No & No & 2 & 1.44 & 1.44 \\
\hline 25 & 74 & $\mathrm{M}$ & $\mathrm{M}$ & 2 & Prostate ca & 2 & 2 & 2 & 2 & No & 1 & No & 1 & No & 3.76 & 4.35 & 4.64 \\
\hline & & & & & & & & & & & & & & & 4.29 & 4.78 & 4.78 \\
\hline 26 & 38 & $\mathrm{~F}$ & $\mathrm{M}$ & 5 & Breast ca & No & No & 5 & 5 & No & No & 3 & 1 & No & 1.82 & 1.82 & 1.82 \\
\hline & & & & & & & & & & & & & & & 1.39 & 1.29 & 1.29 \\
\hline & & & & & & & & & & & & & & & 1.48 & 1.41 & 1.41 \\
\hline & & & & & & & & & & & & & & & 1.50 & 1.50 & 2.35 \\
\hline & & & & & & & & & & & & & & & 1.48 & 1.48 & 1.56 \\
\hline 27 & 33 & $\mathrm{~F}$ & $\mathrm{M}$ & 1 & Thyroid ca & 1 & 1 & 1 & 1 & No & No & 1 & 1 & No & 3.65 & 4.06 & 4.06 \\
\hline 28 & 74 & $\mathrm{M}$ & $\mathrm{M}$ & 1 & Lung ca & No & 1 & No & 1 & No & No & 1 & 1 & No & 2.75 & 3.20 & 3.42 \\
\hline 29 & 43 & $\mathrm{~F}$ & $\mathrm{M}$ & 1 & Thyroid ca & 1 & 1 & No & 1 & No & 1 & 1 & 1 & No & 1.29 & 1.29 & 2.25 \\
\hline 30 & 76 & $\mathrm{M}$ & $\mathrm{M}$ & 1 & Prostate ca & 1 & 1 & 1 & 1 & No & 1 & 1 & 1 & 1 & 3.42 & 3.42 & 3.80 \\
\hline 31 & 45 & $\mathrm{~F}$ & $\mathrm{M}$ & 1 & Thyroid ca & 1 & 1 & 1 & 1 & No & No & 1 & 1 & No & 4.30 & 4.30 & 4.53 \\
\hline 32 & 41 & $\mathrm{~F}$ & $\mathrm{M}$ & 1 & Thyroid ca & No & 1 & 1 & 1 & No & No & 1 & 1 & No & 4.25 & 4.25 & 4.47 \\
\hline 33 & 19 & $\mathrm{M}$ & $\mathrm{M}$ & 1 & Lymphoma & 1 & 1 & 1 & 1 & No & No & No & No & No & 4.71 & 4.71 & 5.23 \\
\hline 34 & 32 & $\mathrm{~F}$ & $\mathrm{M}$ & 2 & Thyroid ca & 2 & 2 & 1 & 2 & 1 & No & No & No & No & 3.07 & 3.56 & 4.29 \\
\hline & & & & & & & & & & & & & & & 3.70 & 3.70 & 3.90 \\
\hline 35 & 31 & $\mathrm{~F}$ & $\mathrm{M}$ & 1 & Thyroid ca & 1 & 1 & 1 & 1 & No & 1 & 1 & 1 & No & 1.31 & 1.31 & 1.31 \\
\hline 36 & 40 & $\mathrm{~F}$ & $\mathrm{M}$ & 1 & Thyroid ca & 1 & 1 & No & 1 & No & No & No & No & No & 3.77 & 3.77 & 4.34 \\
\hline 37 & 41 & $\mathrm{~F}$ & M & 1 & Lung ca & No & 1 & No & 1 & No & No & 1 & 1 & No & 1.43 & 1.43 & 1.54 \\
\hline 38 & 33 & $\mathrm{M}$ & $\mathrm{M}$ & 1 & Lung ca & No & 1 & No & 1 & No & No & No & 1 & No & 3.84 & 3.60 & 4.21 \\
\hline 39 & 71 & $\mathrm{M}$ & $\mathrm{M}$ & 2 & Prostate ca & 2 & 2 & 1 & No & 1 & 1 & No & 1 & No & 3.02 & 3.23 & 3.45 \\
\hline & & & & & & & & & & & & & & & 4.27 & 4.27 & 4.27 \\
\hline 40 & 35 & $\mathrm{~F}$ & $\mathrm{M}$ & 1 & Thyroid ca & No & 1 & No & 1 & No & No & 1 & 1 & No & 4.02 & 4.02 & 4.23 \\
\hline 41 & 68 & $\mathrm{M}$ & $\mathrm{M}$ & 5 & Prostate ca & 5 & 5 & 5 & 5 & No & No & 1 & 1 & No & 3.93 & 4.26 & 4.26 \\
\hline & & & & & & & & & & & & & & & 3.58 & 3.79 & 4 \\
\hline & & & & & & & & & & & & & & & 4.02 & 4.51 & 4.75 \\
\hline & & & & & & & & & & & & & & & 4.84 & 4.84 & 5.43 \\
\hline & & & & & & & & & & & & & & & 2.84 & 3.06 & 3.38 \\
\hline 42 & 21 & $\mathrm{M}$ & $\mathrm{M}$ & 1 & Lymphoma & No & 1 & 1 & 1 & No & No & No & No & No & 2.34 & 2.52 & 2.89 \\
\hline 43 & 43 & $\mathrm{~F}$ & $\mathrm{M}$ & 1 & Thyroid ca & No & 1 & No & 1 & No & No & 1 & 1 & No & 3.01 & 3.36 & 3.36 \\
\hline 44 & 41 & $\mathrm{~F}$ & M & 1 & Thyroid ca & No & 1 & No & 1 & No & No & 1 & 1 & No & 2.03 & 1.89 & 2.03 \\
\hline
\end{tabular}

P.Noa: Numbers of patients, Gb: Gender, Fc:Female, Md: Male, Me/Bf: Malignant/Benign, Fx(n)g: Number of fractures, High SI on T2-WIh: High signal intensity on T2weighted image, High SI on T2-SPIR1: High signal intensity on T2- SPIR sequence, Low SI T1-WIj: Low signal intensity on T1-weighted image, CEk: contrast enhancement, BEl: Band like bone marrow edema, PEm: Involvement of posterior vertebral elements, TCinvn: Involvement of total vertebral corpus, Convo:Convexity of posterior vertebral corpus wall, STp: Associated soft tissue, NADCr: Normalized apparent diffusion coefficient, OPs: Osteoporosis, ca: cancer, Ewing: Ewing sarcoma, MM: multiple myeloma. 
quantitative measurements. High signal intensity on DWI and low signal intensity on ADC map exhibit restriction of diffusion $[1,5-13]$. Degree of DWI is directly related to b-value. The b-value depends on the strength of the gradient, duration of gradient and time between two gradients. Different b-values are mostly achieved due to alterations in strength of gradient. Higher b-value provides stronger diffusion effects and more apparent visualization of diffusion restriction, but decreased signal-to-noise ratio [8, 9]. Therefore, DWI with low (200 $\mathrm{s} / \mathrm{mm} 2)$, intermediate $(400 \mathrm{~s} / \mathrm{mm} 2)$ and high $(600 \mathrm{~s} / \mathrm{mm} 2)$ b-values was performed in our study group. Although DWI is frequently performed for evaluation of acute cerebral ischemia, it is recommended to use for other diseases of different organs especially when there is a difficulty in differential diagnosis. It is known that MRI combined with DWI increases diagnostic accuracy [1, 5-13]. Increased cellularity due to tumor shows restricted diffusion. Bone marrow edema can be excluded on
DWI and ADC map because of non-restriction of diffusion [12].

A number of studies have investigated the diagnostic performance of DWI on differential diagnosis of benign and malignant vertebral lesions and fractures. Castillo et al [14] indicated that qualitative findings of DWI at $b=165 \mathrm{~s} / \mathrm{mm} 2$ had no advantage in the detection of vertebral metastases compared to $\mathrm{T} 1$-weighted sequences in 15 patients. In contrast, qualitative findings of DWI were found valuable in some previous studies [15-17]. The results of these previous studies and ours were summarized in Table 3. In another study, Hamimi et al. [7] demonstrated that osteoporotic fractures $(n=80)$ generally show water line sign and sharp wedging whereas malignant fractures $(\mathrm{n}=70)$ frequently have pedicle involvement, homogenous low signal intensity on $\mathrm{T} 1$-weighted sequence and restricted diffusion. However, there was no quantitative assessment of ADC value [7, 14-15]. Similar qualitative findings were observed in our malignant group.

Table 2 Mean ADC and normalized ADC values of benign and malignant thoracolumbar vertebral fractures according to different b-values.

\begin{tabular}{|c|c|c|c|c|}
\hline & \multicolumn{2}{|c|}{ Mean ADC values $(\mathrm{x} 10-3 \mathrm{~mm} 2 / \mathrm{s})$} & \multicolumn{2}{c|}{ Normalized ADC valuesa } \\
\hline & Benign $(\mathrm{n}=22)$ & Malignant $(\mathrm{n}=50)$ & Benign $(\mathrm{n}=22)$ & Malignant $(\mathrm{n}=50)$ \\
\hline & $( \pm$ SDab) $(95 \% \mathrm{CI})$ & $( \pm \mathrm{SD})(95 \% \mathrm{CI})$ & $( \pm$ SD) $(95 \% \mathrm{CI})$ & $( \pm$ SD) $(95 \% \mathrm{CI})$ \\
\hline $\mathrm{b}=200 \mathrm{~s} / \mathrm{mm}^{*}$ & $4.37 \pm 1.13(3.90-4.85)$ & $1.29 \pm 0.54(1.14-1.44)$ & $12.78 \pm 3.90(11.14-14.41)$ & $3.06 \pm 1.07(2.76-3.36)$ \\
\hline $\mathrm{b}=400 \mathrm{~s} / \mathrm{mm}^{*}$ & $3.98 \pm 0.83(3.62-4.32)$ & $1.34 \pm 0.59(1.18-1.51)$ & $11.53 \pm 2.66(10.42-12.65)$ & $3.14 \pm 1.17(2.82-3.47)$ \\
\hline $\mathrm{b}=600 \mathrm{~s} / \mathrm{mm}^{*}$ & $4.34 \pm 1.13(3.86-4.81)$ & $1.44 \pm 0.61(1.27-1.60)$ & $12.65 \pm 3.84(11.05-14.26)$ & $3.40 \pm 1.24(3.05-3.74)$ \\
\hline
\end{tabular}

aNormalized ADC values: ratios of mean ADC value in vertebral fracture to mean ADC value in adjacent normal vertebral bone marrow abSD: standard deviation, cCI: confidence interval

*Mann Whitney U Test, $\mathrm{p}<0.05$ was used to determine statistical significance between benign and malignant group.

Table 3 Diagnostic performance results of qualitative findings on DWI in previous studies and ours

\begin{tabular}{|c|c|c|c|c|c|c|}
\hline Studies & No of lesions & $\begin{array}{l}\text { Sensitivity } \\
(\%)\end{array}$ & $\begin{array}{l}\text { Specificity } \\
(\%)\end{array}$ & $\begin{array}{l}\text { PPV } \\
(\%)\end{array}$ & $\begin{array}{l}\text { NPV } \\
(\%)\end{array}$ & $\begin{array}{l}\text { Accuracy } \\
(\%)\end{array}$ \\
\hline Our study & 75 & 94 & 91 & 96 & 87 & 93 \\
\hline Bhugaloo15 et al. & 68 & 87 & 92 & 90 & 90 & - \\
\hline Pozzi16 et al. & 33 & 95.6 & 90 & 95.6 & - & - \\
\hline Abowarda17 et al. & 68 & 86 & 91 & 89 & 90 & - \\
\hline
\end{tabular}

PPV: Positive predictive value, NPV: Negative predictive value

Previous studies have also suggested that quantitative evaluation of DWI is necessary and lower ADC values $(1.0 \pm 0.32$ $-1.31 \pm 0.36 \times 10-3 \mathrm{~mm} 2 / \mathrm{s}$ ) were reported in malignant vertebral fractures with b-value of $1000 \mathrm{~s} / \mathrm{mm} 2$ [17-18]. Fawsy et al. [19] performed DWI at two b-values of 500 and 800 and found mean ADC values of $1.21 \pm 1.94 \times 10-3 \mathrm{~mm} 2 / \mathrm{s}$ for benign fractures and $0.69 \pm 0.92 \times 10-3 \mathrm{~mm} 2 / \mathrm{s}$ for malignant ones. Zhou et al [20] evaluated mean ADC value with b-values of 0 and $250 \mathrm{~s} /$ $\mathrm{mm} 2$ for metastases $(\mathrm{n}=15)(1.9 \pm 0.3 \times 10-3 \mathrm{~mm} 2 / \mathrm{s})$ was found significantly lower than that of benign ones $(n=12)(3.2 \pm 0.5 x$ $10-3 \mathrm{~mm} 2 / \mathrm{s}$ ). Similar results were found in ours with different b-values.

Padhani et al [3] calculated that maximum mean ADC value in malignant tumors $(\mathrm{n}=33)$ was $1.4 \times 10-3 \mathrm{~mm} 2 / \mathrm{s}$ with $\mathrm{b}$-values of 50 and 800 or $900 \mathrm{~s} / \mathrm{mm} 2$, but they compared only malignant lesions $(n=33)$ and normal bone marrow $(n=16)$ instead of benign lesions or fractures [3]. In our study, maximum mean ADC value of malignant fractures was higher with $1.92 \times 10-3$ $\mathrm{mm} 2 / \mathrm{s}$ at b-value of $200 \mathrm{~s} / \mathrm{mm} 2$.

In meta-regression analyses, Suh et al [13] found high sensitivity (92\%) and specificity (91\%) ratios of ADC values for differential diagnosis of benign and malignant vertebral fractures. Pozzi et al. [16] mentioned that mean ADC value of malignant fractures $(1.241 \pm 0.4 \times 10-3 \mathrm{~mm} 2 / \mathrm{s})$ was higher than osteoporotic fractures $(0.646 \pm 0.368 \times 10-3 \mathrm{~mm} 2 / \mathrm{s})$ at $b=800$ $\mathrm{s} / \mathrm{mm} 2$. In another study, they also supported these findings with accuracy ratio of $76 \%$ for DWI with ADC measurement at $\mathrm{b}$ values of 0 and $1000 \mathrm{~s} / \mathrm{mm} 2$ [18]. They estimated mean ADC values of malignant primary tumors as $1.00 \pm 0.32 \times 10$ $3 \mathrm{~mm} 2 / \mathrm{s}$, bone metastases as $1.02 \pm 0.25 \times 10-3 \mathrm{~mm} 2 / \mathrm{s}$ and benign primary tumors as $1.31 \pm 0.36 \times 10-3 \mathrm{~mm} 2 / \mathrm{s}$ [18]. In our study, only vertebral fractures were included and ADC values were similarly higher in benign group. Additionally, DWI was performed at multiple b-values.

Luo et al [21] reviewed findings of 12 studies for comparison of DWI at standard $(\geq 500 \mathrm{~s} / \mathrm{mm} 2)$ and low $(<500 \mathrm{~s} /$ $\mathrm{mm} 2$ ) b-values in differential diagnosis. They noted that ADC value difference between benign and malignant group was more apparent at low-b-value $(\mathrm{p}<0.05)$. Therefore, they recommended low-b-value DWI $(<500 \mathrm{~s} / \mathrm{mm} 2)$ for differential diagnosis of benign and malignant vertebral fractures [21]. In contrast, no statistical difference was observed in our study between mean 
ADC values at b-values of 200, 400 and $600 \mathrm{~s} / \mathrm{mm} 2$ except for normalized ADC values of malignant group at b-values of 200 and $600 \mathrm{~s} / \mathrm{mm} 2$.

In some previous studies, cut off points for ADC values were also estimated. Dewan et al. [22] found higher mean ADC value in benign lesions with $b$-value of $1000 \mathrm{~s} / \mathrm{mm} 2$ than that of malignant ones $(\mathrm{p}<0.05)$. With a cut off ADC value of $1.21 \times 10-3 \mathrm{~mm} 2 / \mathrm{s}$, the sensitivity of $95.12 \%$, specificity of $92.73 \%$, was obtained in differential diagnosis [22]. Wonglaksanapimon et al. [23] found the accuracy of $89.7 \%$, sensitivity of $85.7 \%$ and specificity of $90.6 \%$ with a cut off ADC value of 0.89 for differentiation malignant $(n=7)$ from benign $(n=32)$ fractures $(p<0.05)$ [23]. Geith et al [24] found that the best diagnostic performance of DWI and ADC measurements is achieved by a combination of b-values of 100, 250, and $400 \mathrm{~s} / \mathrm{mm} 2$ with a cut off ADC value of $<1.7 \times 10-3 \mathrm{~mm} 2 / \mathrm{s}$ for differential diagnosis of acute benign $(n=26)$ and malignant vertebral fractures $(n=20)($ sensitivity, 85\%; specificity, 84.6\%; PPV, 81.0\%; NPV, 88.0\%) [17]. In our study, a cut off value couldn't be estimated because there was a gap between maximum mean $\mathrm{ADC}$ value of malignant fractures and minimum mean ADC value of benign fractures. Additionally, our maximum mean ADC value of malignant fractures was near to their cut off value $(1.7 \times 10-3 \mathrm{~mm} 2 / \mathrm{s})$ with $1.92 \times 10-3 \mathrm{~mm} 2 / \mathrm{s}$ at b-value of $200 \mathrm{~s} / \mathrm{mm} 2$.

We emphasized that some MRI features like low signal intensity on T1-weighted sequence, total vertebral corpus involvement, contrast enhancement, associated soft tissue component and involvement of posterior elements were strongly associated with malignant fractures. We thought that differential diagnosis can be easier with quantitative measurements of mean ADC and normalized ADC values. Malignant vertebral fractures had lower mean ADC and normalized ADC values compared to benign ones.

One of the limitations in our retrospective study was lack of histopathological results in benign fractures. Vertebral fractures without clinical and radiological progression at least 6 months of follow-up were classified as benign. The other limitation is heterogenity of our sample with the etiology of osteoporosis, trauma, primary malignant tumor and metastases secondary to different malignancies. This heterogenity can cause different diffusion behavior and signal characteristics due to content of tissue.

MRI combined with DWI is a problem solving modality especially in geriatric oncology patients. Performing DWI at least two b-values including b-value of $200 \mathrm{~s} / \mathrm{mm} 2$, quantitative evaluation on ADC map and estimation of normalized ADC value for optimization of data are recommended for differential diagnosis of benign and malignant vertebral fractures.

Disclosures: There is no conflict of interest for all authors.

\section{References}

1. Filograna L, Magarelli N, Cellini F, Manfrida S, Leone A, Colosimo C, et al. Diffusion weighted imaging (DWI) and apparent diffusion coefficient (ADC) values for detection of malignant vertebral bone marrow lesions. Eur Rev Med Pharmacol Sci. 2018; 22(3):590-597.

2. Takigawa T, Tanaka M, Sugimoto Y, Tetsunaga T, Nishida K, Ozaki T. Discrimination between malignant and benign vertebral fractures using magnetic resonance imaging. Asian Spine J. 2017; 11(3):478-483.

3. Padhani AR, van Ree K, Collins DJ, D'Sa S, Makris A. Assessing the relation between bone marrow signal intensity and apparent diffusion coefficient in diffusion-weighted MRI. AJR Am J Roentgenol. 2013; 200(1):163-70.

4. Torres C, Hammond I. Computed tomography and magnetic resonance imaging in the differentiation of osteoporotic fractures from neoplastic metastatic fractures. J ClinDensitom. 2016; 19(1):63-9.

5. Lee DH, Nam JK, Jung HS, Kim SJ, Chung MK, Park SW. Does T1- and diffusion-weighted magnetic resonance imaging give value-added than bone scintigraphy in the follow-up of vertebral metastasis of prostate cancer? InvestigClin Urol. 2017; 58(5):324-330.

6. Mauch JT, Carr CM, Cloft H, Diehn FE. Review of the imaging features of benign osteoporotic and malignant vertebral compression fractures. AJNR Am J Neuroradiol. 2018; 18. doi: 10.3174/ajnr.A5528.

7. Hamimi A, Kassab F, Kazkaz G. Osteoporotic or malignant vertebral fracture? This is the question. What can we do about it? Egypt J RadiolNucl Med.2015; 46: 97-103.

8. Koh DM, Collins DJ. Diffusion-weighted MRI in the body: applications and challenges in oncology. AJR Am J Roentgenol. 2007; 188(6):1622-35.

9. Dietrich O, Biffar A, Baur-Melnyk A, Reiser MF. Technical aspects of MR diffusion imaging of the body. Eur J Radiol. 2010; 76(3):314-22.

10. Dietrich O, Geith T, Reiser MF, Baur-Melnyk A. Diffusion imaging of the vertebral bone marrow. NMR Biomed. 2017; 30(3).

11. Matrawy KA, El-Nekeidy AA, El-Sheridy H. Atypical hemangioma and malignant lesions of spine: Can diffusion weighted magnetic resonance imaging help to differentiate? Egypt J Radiol Nucl Med. 2013; 44:259-263.

12. Caranci F, Tedeschi E, Ugga L, D’Amico A, Schipani S, Bartollino S, et al. Magnetic resonance imaging correlates of benign and malignant alterations of the spinal bone marrow. Acta Biomed. 2018; 19; 89(1-S):18-33.

13. Suh CH, Yun SJ, Jin W, Lee SH, Park SY, Ryu CW. ADC as a useful diagnostic tool for differentiating benign and malignant vertebral bone marrow lesions and compression fractures: a systematic review and meta-analysis. EurRadiol. 2018; 15. doi: 10.1007/s00330-018-5330-5.

14. Castillo M, Arbalaez A, Simith JK, Fisher LL. Diffusion-weighted MRI offers no advantage over routine noncontrast MR imaging in the detection of vertebral metastases. AJNR 2000; 21:948-953. 15.

15. Bhugaloo A, Abdullah B, Siow Y, Ng Kh. Diffusion weighted MR imaging in acute vertebral compression fractures: differentiation between malignant and benign causes. Biomed Imaging Interv J. 2006; 2(2):e12.doi: 10.2349/biij.2.2.e12.

16. Pozzi G, Garcia Parra C, Stradiotti P, Tien TV, Luzzati A, Zerbi A.Diffusion-weighted MR imaging in differentiation between osteoporotic and neoplastic vertebral fractures. Eur Spine J. 2012; 21 Suppl 1:S123-7.

17. Abowarda MH, Abdel-Rahman HM, Taha MM. Differentiation of acute osteoporotic from malignant vertebral compression 
fractures with conventional MRI and diffusion MR imaging. Egypt J RadiolNucl Med.2016; 48:207-213.

18. Pozzi G, Albano D, Messina C, Angileri SA, Al-Mnayyis A, Galbusera F, et al. Solid bone tumors of the spine: Diagnostic performance of apparent diffusion coefficient measured using diffusion-weighted MRI using histology as a reference standard. J MagnReson Imaging. 2018; 47(4):1034-1042.

19. Fawzy F, Tantawy HI, Ragheb A, Hashem AS. Diagnostic value of apparent diffusion coefficient to differentiate benign from malignant vertebral bone marrow lesions. Egypt J RadiolNucl Med.2013; 44:265-271.

20. Zhou JX, Leeds EN, McKinnon CG, Kumar JK. Characterization of benign and metastatic vertebral compression fractures with quantitative diffusion MR imaging. AJNR 2002; 23:165-170.

21. Luo Z, Litao L, Gu S, Luo X, Li D, Yu L, et al. Standard-b-value vs low-b-value DWI for differentiation of benign and malignant vertebral fractures: a meta-analysis. Br J Radiol. 2016; 89(1058):20150384.

22. Dewan KA, Salama AA, El habashyHM, Khalil AE.Evaluation of benign and malignant vertebral lesions with diffusion weighted magnetic resonance imaging and apparent diffusioncoefficient measurements.Egypt J RadiolNucl Med. 2015; 46:423-433.

23. Wonglaksanapimon S, Chawalparit O, Khumpunnip S, Tritrakarn SO, Chiewvit P, Charnchaowanish P. Vertebral body compression fracture: discriminating benign from malignant causes by diffusion-weighted MR imaging and apparent diffusion coefficient value. J Med Assoc Thai. 2012; 95:81-87.

24. Geith T, Schmidt G, Biffar A, Dietrich O, Duerr HR, ReiserM, et al.Quantitative evaluation of benign and malignant vertebral fractures with diffusion-weighted MRI: what is the optimum combination of $b$ values for ADC-based lesion differentiation with the single-shot turbo spin-echo sequence? AJR Am J Roentgenol. 2014; 203(3):582-8.

How to cite this article: Fatma Kulali. Quantitative evaluation of diffusion-weighted imaging with multiple b-values in vertebral fractures. J Clin Med Kaz. 2018; 3(49):35-41 